\title{
Stability and Persistence of Synchronization in a System with a Diffusive-Time-Lag Coupling
}

\author{
Adu A.M. Wasike
}

School of Mathematics, University of Nairobi, P.O. Box 30197- 00100 Nairobi, Kenya, Email: aduwasike@yahoo.com.

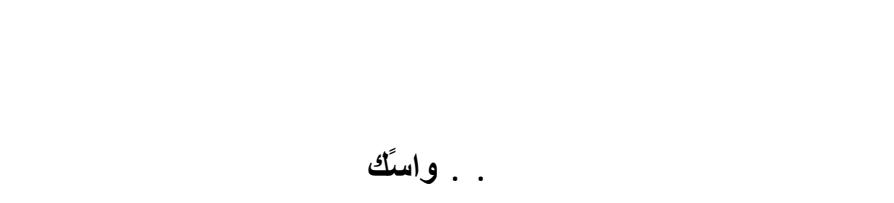

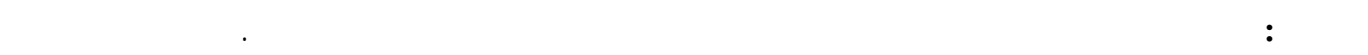

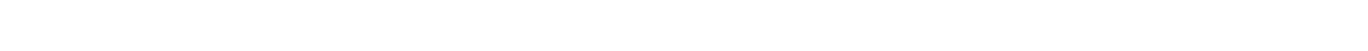
التوافق و شباته.

\begin{abstract}
We study synchronization in the framework of invariant manifold theory for systems with a time lag. Normal hyperbolicity and its persistence in infinite dimensional dynamical systems in Banach spaces is applied to give general results on synchronization and its stability.
\end{abstract}

KEYWORDS: Normal hyperbolicity; Synchronization; Robustness; Lyapunov numbers.

\section{Introduction}

Synchronization phenomena of oscillators and diffusively coupled oscillators has been a subject of great Dinterest by physicist, engineers, and mathematicians, see for instance (Afraimovich, et.al., 1986; Chow and Liu, 1997; Fujisaka and Yamada, 1983; Hale, 1997; Wasike, 2002; Wasike, 2003; Wasike and Rotich (2007).

Basic to the study of synchronization, two fundamental questions are of interest. The first is to do with the stability of the synchronization state of the system and the second is its robustness. Robustness of the synchronization state is its ability to be insensitive to small perturbations in the system that generates it. The two questions have been done for diffusively coupled systems without a time lag in the coupling. Normal hyperbolicity and the generalized Lyapunov exponents have been used to establish conditions for the stability and persistence of synchronization manifold for lattice dissipative systems each with a compact global attractor, see for example (Chow and Liu, 1997; Wasike, 2002; Wasike, 2003; Josica, K, 2000; Wasike and Rotich, 2007).

The subject of stability and persistence of synchronization in a system with a diffusive-time-lag coupling has received less attention. Grasman and Jansan (1979) have studied synchronization in oscillators coupled in only one variable with a time lag in which phase differences have been used to detect synchrony. Numerical 


\section{ADU A.M. WASIKE}

results have also been used to determine the Lyapunov exponents in order to exhibit stability of the synchronization manifold, see for instance (Pyragas, 1998; Rossoni, 2005). To the best of my knowledge, no rigorous mathematical results in the framework of robustness of invariant manifolds for delay differential equations have been reported.

This is how we approach this problem. We set the problem in the framework of dynamical systems and then consider the two aspects of synchronization based on invariant manifold theory for systems with a time lag. For the theory of invariant manifold for systems with a time lag, see for instance Halanay (1967), Kurzweil (1967). Normal hyperbolicity is exactly the right condition for persistence of invariant manifolds (see Bates et. al., 1998). In particular, we shall apply the theory of normally hyperbolic invariant manifolds for semiflows in Banach spaces as defined in Bates et al., $(1998,1999,2000)$. This approach will enable us to compare the rate of growth in the transversal direction to the synchronization manifold and that along the manifold.

This is our road map. Most of the definitions and terminologies will be given in $\S 2$ while in $\S 3$ we present the main results on the robustness of a synchronization manifold where normal and tangential growth rates are compared. In $\S 4$ we give an example of an all-to-all coupled system. $\S 5$ is the conclusion.

\section{Definitions and terminologies}

Let $X=C\left([-r, 0] \mathbb{R}^{n} \times \mathbb{R}^{m}\right)$ be the space of continuous functions from $[-r, 0]$ to $\mathbb{R}^{n} \mathrm{x} \mathbb{R}^{m}$ endowed with the usual supremum topology. Consider the following system

$$
\begin{aligned}
& \dot{x}(t)=f_{1}\left(x_{t}, y_{t}\right), \\
& \dot{y}(t)=f_{2}\left(x_{t}, y_{t}\right),
\end{aligned}
$$

where

$x \in C\left([-r, 0], \mathbb{R}^{n}\right), y \in C\left([-r, 0], \mathbb{R}^{m}\right), x_{t}(\theta)=x(t+\theta), y_{t}(\theta)=y(t+\theta), \theta \in[-r, 0]$,

$f_{1}: X \rightarrow \mathbb{R}^{n}, f_{2}: X \rightarrow \mathbb{R}^{m}$ are continuous functions defined on $X$ to $\mathbb{R}^{n}$ and $\mathbb{R}^{m}$, respectively.

Let

$$
f\left(x_{t}, y_{t}\right)=\left(\begin{array}{c}
f_{1}\left(x_{t}, y_{t}\right) \\
f_{2}\left(x_{t}, y_{t}\right)
\end{array}\right) \text {. }
$$

For any $\varphi=\left(\varphi^{c}, \varphi^{S}\right) \in X$, equation (1) has a unique solution $(x(t, \varphi), y(t, \varphi))$ which is continuous for $t \geq-r$ and coincides with $\varphi$ on $[-r, 0]$. Let $(T(t) \varphi)(\theta)=\left(x_{t}(\theta, \varphi)\right), \theta \in[-r, 0]$ and assume all solutions are uniquely defined for $t \geq 0$, then $T(t)$ is a $C^{0}$ - semigroup on $X$. Suppose that the system in equation (1) is dissipative, then there exists a global attractor $A$.

The following definition is motivated by the works of Halanay (1967) and also Kurzweil (1967).

2.1 Definition. System (1) is synchronized for $y$ with respect to $x$, if there exists a $C^{1}$ map $H: \mathbb{R}^{m} \rightarrow C$, where $n$ is the dimension of $x$ and $C=C\left([-r, 0], \mathbb{R}^{m}\right)$, with $m$ the dimension of $y$ such that the graph of $H$ denoted graph $(H)$, is invariant and globally attracting. 


\section{STABILITY AND PERSISTENCE OF SYNCHRONIZATION}

In this case, for any $\varphi=\left(\varphi^{c}, \varphi^{s}\right) \in X$ has

$$
\left\|y_{t}(\varphi)-H\left(x_{t}(\varphi)\right)\right\| \rightarrow 0 \text { as } t \rightarrow \infty \text {; }
$$

exponentially. In particular, if $\varphi \in A$, then $\varphi^{S}=H\left(\varphi^{c}\right)$ and $y_{t}(\varphi)=H\left(x_{t}(\varphi)\right)$ for all $t \geq 0$, and this $H$ is unique (see Halanay 1967; p. 209).

In case, $m=n$ equation (1) is mutually synchronized if $H$ is invertible.

For the system with $D=\{(x, y): x=y\}$ invariant, for example, in coupled identical systems or any system symmetric with respect to $D$, the synchronization occurs for $H=I$. In this case, the diagonal in $X$ that consists of the set of functions $\varphi=\left(\varphi^{c}, \varphi^{S}\right)$ for which $\varphi^{c}=\varphi^{S}$ is an invariant set for equation (1).

In many applications, we are interested in local synchronization. We are interested in the local attractivity of graph $(H)$; that is, the graph of $(H)$ is exponentially attracting in the neighbourhood of $\mathrm{D}$,

$$
\left\|y_{t}(\varphi)-H\left(x_{t}(\varphi)\right)\right\| \leq k e^{-\alpha t}\left\|\varphi^{s}-H\left(\varphi^{c}\right)\right\|,
$$

where $k, \alpha$ are positive constants.

2.2 Definition. Suppose equation (1) is locally synchronized with map $H$. The synchronization is $C^{1}$ stable if for any $\in>0$,

there is a $\delta>0$ such that for any $\tilde{f}_{1}, \tilde{f}_{2},\left|\tilde{f}_{1}-\tilde{f}_{1}\right|_{C^{1}}<\delta$ and $\left|\tilde{f}_{2}-\tilde{f}_{2}\right|_{C^{1}}<\delta$, the system

$$
\begin{aligned}
\dot{x}(t) & =\tilde{f}_{1}\left(x_{t}, y_{t}\right) \\
\dot{y}(t) & =\tilde{f}_{2}\left(x_{t}, y_{t}\right),
\end{aligned}
$$

is locally synchronized with map $\tilde{H}$ and $|\tilde{H}-H|_{c_{1}}<\in$ see (Bates et .al ;1998 p. 119).

\section{Main Results: Robustness of a synchronized manifold}

Let us recall some of the invariant manifold theory for infinite dimensional systems in a Banach space. Let $X$ represent a Banach space with norm $|\cdot| \cdot$ In subspaces the same norm symbol is used. The notation $\|\cdot\| \cdot$ will be reserved for the linear operator norm

$\|L\| \equiv \sup \{|L x|:|x|=1, x \in D(L)\}, \quad m(L)=\min \{|L x|:|x|=1, x \in D(L)\}$ where $D(L)$ means the domain of the operator $L$.

For any $\varphi \in X$, there is a unique solution $z(t, \varphi)$ to equation (1) that defines a $C^{1}$ semiflow on $X$; that is,

$$
T^{t} \varphi=z(t, \varphi)
$$

is continuous on $[0, \infty) \times X$, and for each $t \geq 0, T^{t}: X \rightarrow X$ is $C^{1}$ and $T^{t} \circ T^{s}(\varphi)=T^{t+s}(\varphi)$ for all $t, s \geq 0$, and $\varphi \in X$. 


\section{ADU A.M. WASIKE}

Suppose, for System (1), there exists a smooth connected invariant manifold $M=\operatorname{graph}(H) \subset X$; that is, $T^{t}(M) \subset M$ for each $t \geq 0$. Consider the linearization of equation (1) along $M$

$$
\dot{z}(t)=A(z(t, \varphi)) z_{t},
$$

where $z(t, \varphi)$ is the solution of Equation (1) with $z(0, \varphi)=\varphi \in M$ and $A(z(t, \varphi))$ is Jacobian of $f$ at $z(t, \varphi)$. Let $D T^{t}(m)$ be the linearized semiflow associated with equation (3). $M$ is said to be normally hyperbolic if the following are satisfied:

(H1) The tangent bundle of $X$ restricted to $M$ splits into two continuous subbundles

$$
\left.T X\right|_{M}=X^{c} \oplus X^{S}
$$

where $X^{c}$ is the tangent bundle of $M$ and $X^{s}$ is transversal to $X^{c}$. The superscripts $c$ and $s$ stand for "center", and "stable", respectively.

(H2) This splitting is invariant under $D T^{t}$; that is, for each $m \in M$ and $t \geq 0$, if $m_{1}=T^{t}(m)$, and $D T_{p}^{t}(m)=\left.D T^{t}(m)\right|_{X_{m}^{p}, p=c, s,}$ then

$$
D T_{p}^{t}(m): X_{m}^{p} \rightarrow X_{m}^{p}
$$

and $D T_{p}^{t}(m)$ is an isomorphism from $X_{m}^{p}$ onto $X_{m_{1}}^{p}$, where $X_{m}^{p}$ denote the fibers for the vector bundles $X^{p}, p=c, s$ at $m \in M$.

(H3) There exists $t_{0} \geq 0$ and $\lambda \in(0,1)$ such that for all $t \geq t_{0}$ and $m \in M$,

$$
\frac{\left\|\left.D T^{t}(m)\right|_{x_{m}^{s}}\right\|}{\min \left\{1, \inf \left\{\left|D T^{t}(m) x^{c}\right|: x^{c} \in X_{m}^{c},\left|x^{c}\right|=1\right\}\right\}}<\lambda .
$$

For this definition see Bates et al., $(1998,1999)$. A few remarks on the above definition of normal hyperbolicity are useful.

Remark. Inequality (4) suggests that near $m \in M, T^{t}$ contracts in the direction of $X_{m}^{s}$, and at a rate greater than that on M. Furthermore (4) suggests that for an invariant splitting to persist we need both

$$
\left\|D T_{s}^{t}(m)\right\|<1
$$

and

$$
\left\|D T_{s}^{t}(m)\right\|<\inf \left\{\left|D T^{t}(m) x^{c}\right|: x^{c} \in X_{m}^{c},\left|x^{c}\right|=1\right\}<1,
$$




\section{STABILITY AND PERSISTENCE OF SYNCHRONIZATION}

to be satisfied, see for instance Bates et al. (1998, pages 124 and 11, respectively).

\subsection{Generalized Lyapunov-type numbers}

For the purpose of calculation it will prove convenient to phrase inequalities (5) and (6) more quantitatively in terms of rates of growth. By the equivalence of norms,

$$
\inf \left\{D T^{t}(m) x^{c}\left|: x^{c} \in X_{m}^{c},\right| x^{c} \mid=1\right\} \leq\left\|D T_{c}^{t}(m)\right\| \text {. }
$$

Thus inequality (6) reduces to

$$
\frac{\left\|D T_{s}^{t}(m)\right\|}{\left\|D T_{c}^{t}(m)\right\|}<1 .
$$

Let us now compare the growth bounds of the two semigroup operators. The growth bound of the linear operator $D T_{c}^{t}(m)$ is a real number $\omega_{0}$ defined by

$$
\omega_{0}=\inf \left\{\omega \mid \exists M_{c} \geq 1 \text { such that }\left\|D T_{c}^{t}(m)\right\| \leq M_{c} e^{\omega t} \text {, for } t \geq 0\right\} .
$$

This is the same as

$$
\omega_{0}=\inf _{t>0} \ln \left\|D T_{C}^{t}(m)\right\|=\lim _{t \rightarrow \infty} \frac{1}{t} \ln \left\|D T_{C}^{t}(m)\right\|,
$$

see for instance Diekmann et al (1995, p. 470). We also know that the $\left\|D T_{s}^{t}(m)\right\|$ satisfies

$$
\left\|D T_{s}^{t}(m)\right\| \leq M_{s} e^{\alpha t}
$$

for $t \geq 0$, where $M_{S}>0$ and $\alpha$ are real constants. Without loss of generality, we can take $M_{C}$ and $M_{S}$ each equal to 1 . This is possible by the use of the next lemma.

Lemma 3.1 The spaces $X_{m}^{c}$ and $X_{m}^{s}$, for all $m \in M$, can be renormed so that in equations (8) and (10) $M_{c}$ and $M_{s}$ can be taken to be 1 .

Proof. Define a new norm $|\cdot|^{\prime}$ on $X_{m}^{s}$ by

$$
|x|^{\prime}=\sup _{t \geq 0} e^{-\alpha t}\left|D T_{s}^{t}(m) x\right| \text { for } x \in X_{m}^{s} .
$$

Clearly, $|x| \leq|x|^{\prime}$ and by equation (10) $|x|^{\prime} \leq M_{s}|x|$, so $|\cdot|$ and $|\cdot|^{\prime}$ are equivalent norms on $X_{m}^{s}$. Let $\|\cdot\| \|^{\prime}$ be the operator norm on $X_{m}^{s}$ equipped with $|\cdot|^{\prime}$. Then for $x \in X_{m}^{s}$ and $\tau \geq 0$

$$
\begin{aligned}
\left|D T_{s}^{t}(m) x\right|^{\prime} & =\sup _{t \geq 0} e^{-\alpha t}\left|D T_{s}^{t}(m) D T_{s}^{T}(m) x\right| \\
& =\sup _{t \geq 0} e^{-\alpha(t+T)}\left|D T_{s}^{t+T}(m) x\right| e^{-\alpha t} \\
& =\sup _{t \geq T} e^{-\alpha t}\left|D T_{s}^{t} x\right| e^{\alpha t} \leq|x|^{\prime} e^{\alpha t} .
\end{aligned}
$$




\section{ADU A.M. WASIKE}

Hence, $\left\|D T_{s}^{t}(m) x\right\|^{\prime} \leq e^{\alpha t}$ for $\tau \geq 0$. The proof for $X_{m}^{c}$ is similar.

We now define some generalized Lyapunov exponents for semiflows.

3.2 Definition. The generalized Lyapunov exponents for $m \in M$ are defined as follows

$$
\alpha(m)=\limsup _{t \rightarrow \infty} \frac{1}{t} \ln \left\|D T_{s}^{t}(m)\right\|
$$

and

$$
\beta(m)=\limsup _{t \rightarrow \infty} \frac{\ln \left\|D T_{s}^{t}(m)\right\|}{\ln m\left(\left\|D T_{c}^{t}(m)\right\|\right)} .
$$

The generalized Lyapunov exponents were introduced for the study of normally hyperbolic invariant manifolds in finite dimensional systems, see for instance (Chow and Liu, 1997; Fenichel, 1971; Hirsch et al., 1977; Wiggins, 1994). No similar results exist for semiflows.

The results we have are:

Theorem 3.3 Consider System (1). Suppose the graph $(\mathrm{H})$ is invariant. If $\alpha(m)<0$ for $m \in$ graph $(H)$, then graph $(H)$ is attracting, and hence equation (1) is locally synchronized.

Theorem 3.4 Suppose that graph $(\mathrm{H})$ is locally synchronized. The synchronization is $C^{1}$ stable if $\alpha(m)<0$ and $\beta(m)<1$ for all $m \in \operatorname{graph}(\mathrm{H})$.

Proof. The proof makes use of equations (9), (10) and Lemma 3.1. We have that

$$
\alpha(m)=\alpha \text { and } \beta(m)=\frac{\alpha}{\omega_{0}} .
$$

For the stability of the synchronized manifold, we require that inequality (5) be satisfied. This requirement translates to $\alpha(m)<0$ for all $m \in M$. For persistence, inequality (7) implies that $\beta(m)<1$.

\section{Example: Synchronization of all-to-all coupled systems}

In this part, the theorems are applied to the study of synchronization of all-to-all coupled systems. Let

$$
\dot{z}(t)=g(z(t)),
$$

$z \in \mathbb{R}^{d}$, be a dissipative processes and $A$ be the attractor. Consider the coupled system

$$
\begin{aligned}
& \dot{z}_{1}(t)=k\left(z_{2}(t-r)-z_{1}(t)\right)+g\left(z_{1}(t)\right), \\
& \dot{z}_{2}(t)=k\left(z_{1}(t-r)-z_{2}(t)\right)+g\left(z_{2}(t)\right) .
\end{aligned}
$$

Let $X=C\left([-r, 0], \mathbb{R}^{d} \times \mathbb{R}^{d}\right)$. Equation (15) subject to $\varphi=\left(\varphi_{1}, \varphi_{1}\right) \in X$ has a unique solution $z_{1}(t, \varphi), z_{2}(t, \varphi) \forall t \geq 0$, which coincides with $\varphi$ on $[-r, 0]$. 


\section{STABILITY AND PERSISTENCE OF SYNCHRONIZATION}

Equation (15) is synchronized if its solution belongs to a compact inertial manifold $M_{k} \subset M$,

$$
M=\left\{\left(\varphi_{1}, \varphi_{1}\right) \in X \mid \varphi_{1}=\varphi_{1}\right\} .
$$

To show synchronization, we show that $M$ is attracting; that is, $z_{1}(t, \varphi)-z_{2}(t, \varphi) \rightarrow 0$ as $t \rightarrow \infty$. For this purpose, we need to study the semiflow tangential and transversal to $M$. We thus make the change of variables

$$
w(t)=\frac{z_{1}(t)-z_{2}(t)}{2}, y(t)=\frac{z_{1}(t)+z_{2}(t)}{2} .
$$

With the transformation (16) in equation (15), we get

$$
\begin{aligned}
& \dot{w}(t)=-k[w(t-r)+w(t)]+\frac{1}{2}[g(w(t)+y(t))-g(y(t)-w(t))], \\
& \dot{y}(t)=k[y(t-r)-y(t)]+\frac{1}{2}[g(w(t)-y(t))+g(y(t)+w(t))] .
\end{aligned}
$$

Synchronization is equivalent to the fact that $\{w(t, \varphi)=0\}$ is attracting. By the linearization of equation (15) along $\{w(t, \varphi)=0\}$; that is, along $(w(t), y(t)) \in M_{k} \subset M$, equation (17) becomes

$$
\begin{aligned}
& \dot{w}(t)=-k[w(t-r)+w(t)]+D_{z} g\left(y_{0}(t)\right) w(t), \\
& \dot{y}(t)=k[y(t-r)-y(t)]+D_{z} g\left(y_{0}(t)\right) y(t),
\end{aligned}
$$

where $D_{z} g\left(y_{0}(t)\right)$ is the Jacobian of $g(z(t))$ at $\left(y_{0}(t)\right)$, the solution is obtained from

$$
\dot{y}(t)=k[y(t-r)-y(t)]+g(y(t)) .
$$

Notice that there is a continuous invariant splitting $X^{S} \oplus X^{c}$, where

$$
\begin{aligned}
& X^{s}=\{(w(t, \varphi), y(t, \varphi)) \mid y(t, \varphi)=0\}, \\
& X^{c}=\{(w(t, \varphi), y(t, \varphi)) \mid w(t, \varphi)=0\} .
\end{aligned}
$$

Let us now look at the attractivity of $X^{s}$. Clearly $W(t)=I_{d} e^{\lambda^{s} t}$, where $\lambda^{s}$ satisfies the characteristic equation $h^{s}(\lambda):=\lambda+k\left(1+e^{-\lambda r}\right)=0$, is the fundamental matrix solution of the first equation in equation (18); that is,

$$
\dot{W}(t)=-k[W(t-r)+W(t)] .
$$

By the variation-of-constants formula, the solution $w(t, \varphi)$ to the first equation in equation (18) is given by

$$
w(t, \varphi)=W(t)\left[W^{-1}(0) \varphi(0)+\int_{0}^{t} W^{-1}(\tau) D_{z} g\left(y_{0}(\tau)\right) w(\tau) d \tau\right] .
$$

By the Gronwall's Inequality

$$
w(t, \varphi) \leq \varphi(0) e^{\int_{0}^{t}\left(D_{z} g\left(y_{0}(\tau)\right)-\lambda^{S} I_{d}\right) d \tau} .
$$


Let $\lambda_{M}$ and $\lambda_{m}$ be the maximum and minimum Lyapunov exponents of the trajectory defined by $\dot{y}(t)=D_{z} g\left(y_{0}(t)\right) y(t)$. Then for $w(t, \varphi) \rightarrow 0$ as $t \rightarrow \infty$, we require

$$
\max \left\{\lambda_{M}+\operatorname{Re}\left(\lambda^{s}\right)\right\}<0 .
$$

Since all roots of $h^{s}(\lambda)=0$ for $k>0$ have negative real parts (see for instance Bose; 1986), this would mean

$$
\lambda_{M}<\max \left|\operatorname{Re}\left(\lambda^{s}\right)\right|=k, k>\lambda_{M} .
$$

Indeed

$$
\lambda^{s}=-k+i / r(\pi / 2+2 m \pi), k r e^{k r}=\pi / 2+2 m \pi, m=0,1,2, \ldots \ldots
$$

By Theorem 3.2 $\{w(t, \varphi)=0\}$ is attracting and the coupled system is locally synchronized.

The solution $y(t, \varphi)$ of the second equation in equation (18) is given by

$$
y(t, \varphi)=Y(t)\left[Y^{-1}(0) \varphi(0)+\int_{0}^{t} Y^{-1}(\tau) D_{z} g\left(y_{0}(\tau)\right) y(\tau) d \tau\right] \text { for } t \geq 0,
$$

where $Y(t)=I_{d} e^{\lambda^{c} t}, \lambda^{c}$ satisfies the characteristic equation $h^{c}(\lambda):=\lambda+k\left(1-e^{-\lambda r}\right)=0$, is the fundamental matrix solution of the second equation in (18) that is,

Indeed

$$
\dot{Y}(t)=k[Y(t-r)-Y(t)]
$$

$$
\lambda^{c}=-k+i / r(\pi / 2+(2 m+1) \pi), k r e^{k r}=\pi / 2+(2 m+1) \pi, m=0,1,2, \ldots \ldots
$$

For persistence we have

$$
\max \left\{\lambda_{M}-\operatorname{Re}\left(\lambda^{s}\right)\right\} \leq \min \left\{\lambda_{m}-\operatorname{Re}\left(\lambda^{c}\right)\right\},
$$

where $\lambda^{c}$ satisfies the characteristic equation $h^{c}(\lambda)=0$ whose roots except $\lambda=0$ lie to the left of the complex plane(see for instance Bose, 1986). Equation (19) gives $k>\lambda_{M}-\lambda_{m}$. By Theorem 3.3, the synchronization manifold is robust.

\section{Conclusion}

Time delay in the coupling does not always destabilize synchronization states of similar systems with delay $r=0$.

\section{References}

AFRAIMOVICH, V.S, VERCHEV, N.N. and RIABONVICH, M.I. 1986. Stochastic Synchronization of oscillations in dissipative systems. Radio Phy. Quantum Electron. 29: 31-45.

BATES, P.W., LU, K., ZENG, C. 1998. Existence and persistence of invariant manifold for semiflows in Banach spaces, Mem. Amer. Math. Soc. 135(645): 305-317. 


\section{STABILITY AND PERSISTENCE OF SYNCHRONIZATION}

BATES, P.W., LU, K., ZENG, C. 1999. Persistence of overflowing Manifolds for semiflows, Comm. Pure Appl. Math. LII: 983-1046.

BATES, P.W., LU, K., ZENG, C. 2000. Invariant Foliations near Normally Hyperbolic Invariant Manifolds For Semiflows, Trans. Amer. Math. Soc. 352(10): 4641-4676.

BOSE, F.G. 1986. Stability Conditions for the General Linear Difference-Differential Equation with Constant Coefficients and One Constant Delay. J. Math. Anal. Appl. 140:136-176.

CHOW, S.N. and LIU, W. 1997. Synchronization, stability and normal hyperbolicity. Resenhas IME-USP\}. 3: 139-158.

CHOW, S.N. 2000. Lattice Dynamical Systems, Dynamical Systems Springer. 1822: 1-99.

DIEKMANN, O., VAN GILS, S. A.,VERDUYN LUNEL, S.M. and WALTHER, H.-O. 1995. Delay Equations. Springer-Verlag, Berlin, Heidelberg, New York.

FENICHEL, N. 1971. Persistence and smoothness of invariant manifolds for flows. Ind, Univ. Math. J., 21(3): 193-225.

FUJISAKA, H. and YAMADA, T. 1983. Stability Theory of Synchronized Motion in Coupled-Oscillator System. Prog. Theor. Phys. 69: 32-47.

GRASMAN, J., and JANSAN, M.J.W. 1979. Mutually synchronized relaxation oscillators as prototypes of oscillating systems in biology. J. math. Biology 7: 171-197.

HALANAY, A. 1967. Invariant manifolds for systems with Time lag, Differential Equations and Dynamical systems. Academic Press. 199-213.

HALE, J. 1997. Diffusive coupling, dissipation and synchronization J. Dyn. Differ. Equ. 9:1-52.

HALE, J.K, and VERDUYN LUNEL. 1993. Introduction to functional Differential Equation; Springer-Verlag: New York.

HENRY, D. 1983. Geometric Theory of Semilinear Parabolic Equations, Lecture Notes in Mathematics. No. 840, Springer-Verlag: Berlin.

HIRSCH, M.W., PUGH, C.C., SHUB, M., Invariant manifolds. Lecture Notes in Mathematics Vol 583. Springer-Verlag: Berlin, Heidelberg, New York, 1977.

JOSICA, K. 2000. Synchronization of chaotic systems and invariant manifolds, Nonlinearity 13: 1321-1336.

KURZWEIL, J. 1967. Invariant Manifolds for flows, Differential Equations and Dynamical systems. Academic Press. 431-468.

PYRAGAS, K. 1998. Synchronization of coupled time-delay systems: Analytical estimations Physical Review E. 53(3): 3067-3071.

ROSSONI, E., CHEN, Y., DING, M., FENG, J. 2005. Stability of synchronous oscillations in systems of HH neurons with delayed diffusive and pulsed Coupling. Phys. Review E. 71: 819-823.

WASIKE, A.A.M. 2002. Periodic solutions of Systems of delay differential equations Indian Journal of Mathematics, 44(1): 95-117.

WASIKE, A.A.M. 2003. Synchronization and oscillator death in diffusively coupled lattice oscillators International Journal of Mathematical Science, 2(1): 67-82.

WASIKE, A.A.M., Rotich, K.T. 2007. Synchronization, Persistence in Diffusively coupled Lattice oscillators Sultan Qaboos University Journal of Science, 12(1): 41-52 .

WIGGINS, S. 1994. Normally Hyperbolic Invariant Manifolds in Dynamical Systems. Springer-Verlag: Berlin, Heidelberg, New York.

Received 12 September 2007

Accepted 31 May 2008 